Manuscript Title: Age-specific associations between violence exposure and past 30-day marijuana and alcohol use.

Author List: Jason E. Goldstick PhD ${ }^{1,2}$, Justin Heinze PhD ${ }^{3,4}$, Sarah A. Stoddard PhD ${ }^{5}$, Rebecca M. Cunningham MD ${ }^{1,2,3}$, Marc A. Zimmerman PhD $^{2,3,4}$

${ }^{1}$ Department of Emergency Medicine, University of Michigan, E Medical Center Dr, Ann Arbor, MI, 48109

${ }^{2}$ Injury Prevention Center, University of Michigan, 2800 Plymouth Road, Suite B10-G080, Ann Arbor, MI 48109-2800

${ }^{3}$ University of Michigan Youth Violence Prevention Center, 1415 Washington Heights, Ann Arbor, MI 48109

${ }^{4}$ Department of Health Behavior and Health Education, School of Public Health, University of Michigan, 1415 Washington Heights, Ann Arbor, MI 48109.

${ }^{5}$ Department of Systems, Populations, and Leadership, School of Nursing, University of Michigan, 400 North Ingalls, Ann Arbor, MI 48109.

Corresponding Author: Jason E. Goldstick, Injury Prevention Center, University of Michigan, 2800 Plymouth Road, Suite B10-G080, Ann Arbor, MI 48109-2800

Phone: 734-936-9312, Fax: 734-764-2020, Email: jasoneg@umich.edu

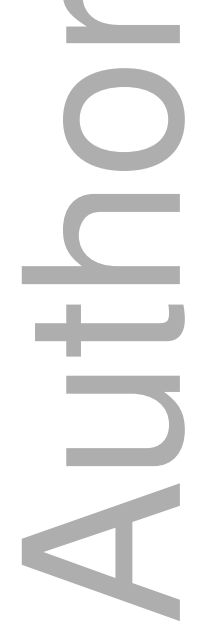

This is the author manuscript accepted for publication and has undergone full peer review but has not been through the copyediting, typesetting, pagination and proofreading process, which may lead to differences between this version and the Version of Record. Please cite this article as doi: $\underline{10.1111 / \text { jora.12399 }}$

This article is protected by copyright. All rights reserved 


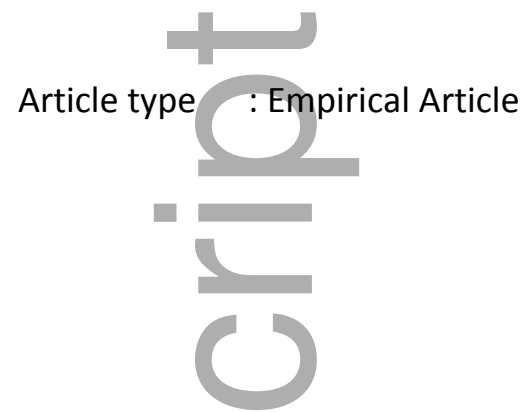

\begin{abstract}
Using data from a cohort study of students at risk for high school dropout, we examined associations between violence exposure and past 30-day alcohol and marijuana use. We used varying-coefficient regression with person-level fixed effects to estimate how those associations changed within-person across ages approximately 14-23. Generally, violence perpetration was most strongly associated with substance use, within-person. Substance use became increasingly associated with both observed violence and violence perpetration during early/middle adolescence; this increase continued longer into development (age 18+) for alcohol use. Across most of the age range studied here, violence victimization was minimally associated with within-person changes in substance use. Results indicate age-specific associations between violence exposure and AOD use, which may be useful for informing prevention strategies.
\end{abstract}

Keywords: marijuana, alcohol, violence

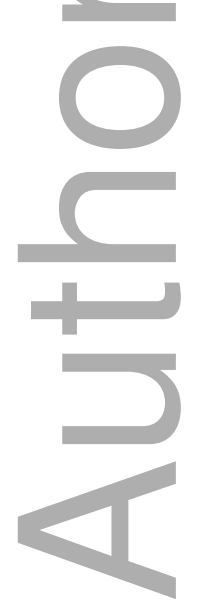


Introduction

Exposure to violence (ETV) is associated with physical and mental health consequences (Olofsson, Lindqvist, Shaw, \& Danielsson, 2012; Springer, Sheridan, Kuo, \& Carnes, 2007), and disproportionately affects low income populations in the United States, acting in tandem with a variety of other stressors to exacerbate health disparities among those living in poor neighborhoods (Sheidow, Henry, Tolan, \& Strachan, 2014). A common correlate of violence exposure is alcohol and other drug (AOD) use (Fagan, Wright, \& Pinchevsky, 2015; Resnick, Ireland, \& Borowsky, 2004; Wright, Fagan, \& Pinchevsky, 2013). The link between ETV and AOD use is complex and can be explained from a number of different angles. For example, there is evidence that AOD use may be acutely implicated in violence perpetration or victimization (Epstein-Ngo et al., 2013; Epstein-Ngo et al., 2014; Giancola, 2002; Stoddard et al., 2015). On the other hand, researchers have found some support for the idea that AOD use occurs in response to violence exposure (McGovern et al., 2009; Vermeiren et al., 2003), suggesting some bi-directionality to the relationship. Regardless of the direction of the relationship, however, the multifaceted transitions inherent to adolescence and emerging adulthood may modulate the ETV/AOD association during that developmental stage. While researchers have established that rates of both substance use (SAMSHA, 2013) and violence exposure (Loeber \& Farrington, 2012; Resnick et al., 2004; Stoddard et al., 2015; Sullivan, Farrell, \& Kliewer, 2006) increase across adolescence, few have focused on the age-specific association between the two. The focus of this study is to estimate how the association between violence exposure and AOD use change across ages 14 to 23 .

Violence Exposure, Psychological Distress, and AOD use

Researchers have consistently identified associations between exposure to violence and negative psychosocial and health outcomes (Boynton-Jarrett, Ryan, Berkman, \& Wright, 2008; Dahlberg \& Potter, 2001; Fuller-Thomson, Roane, \& Brennenstuhl, 2016; Gorman-Smith, Henry, \& Tolan, 2004; Herrenkohl et al., 2000; Schilling, Aseltine, \& Gore, 2007; Goldstick et al, 2017a), and these associations are often conceptualized as mediated by psychological distresses (Deane et al., 2016). In particular, exposure to violence places adolescents at increased risk of internalizing problems, including major depressive disorders and post-traumatic stress disorder (Deane et al., 2016; Sheidow et al., 2014). In addition, many individuals exposed to violence have experienced different types of violence exposure (e.g. both peer and partner violence, and both as perpetrator and victim) (Finkelhor, Ormrod, Turner, \& Hamby, 2005; Kitzmann, Gaylord, Holt, \& Kenny, 2003; Turner, Shattuck, Finkelhor, \& Hamby, 2016). A corollary of the varied forms of violence exposure is the possibility that different exposures confer different health 
VIOLENCE EXPOSURE AND AOD USE ACROSS AGES

risks. In this study we focus on three different types of violence exposures: victimization, perpetration, and direct observation of violence in the individual's community

AOD use is consistently found to be associated with ETV, and there are many possible explanations for that association. For example, the pharmacological effects of alcohol use has been shown to decrease impulse control and increase aggression (Giancola, 2002). More generally, both aggression and victimization have been shown to be more likely on days where substances were used (Epstein-Ngo et al., 2013; Epstein-Ngo et al., 2014; Stoddard et al., 2015). Those observed relationships may also relate to the fact that AOD use sometimes places individuals in situations where violence exposure is more likely, e.g. those necessitating contact with the illegal drug trade (Friedman, Glassman, \& Terras, 2001). On the other hand, AOD use is associated with mental health problems that frequently arise from repeated violence exposure, such as post-traumatic stress disorder (Read, Brown, \& Kahler, 2004; McGovern et al., 2009), major depressive disorder (Agrawal, Lynskey, Madden, Bucholz, \& Heath, 2007), and anxiety disorders (Buckner et al., 2012). Thus, AOD use may arise as a coping strategy for the cumulative stress incurred by frequent exposure to violence (Vermeiren et al., 2003). Alternative theories suggest that the association between certain types of ETV (e.g. aggression) with AOD use may be explained by clustering of problem behaviors (Jessor, 1991). Regardless of the lead-lag relationship of AOD and ETV (i.e., the temporal direction of the causal pathway), developmental specificity in the association may be present. Accordingly, one may be more (or less) prone to aggressive behavior while acutely intoxicated as they age, one may cope differentially with ETV at different ages, and behaviors may cluster differently at different developmental stages. Given the substantial and varied difficulties incurred from AOD using during adolescence and early adulthood, further clarifying the role through understanding those agespecific associations with one of its strongest correlates remains a priority.

Adolescence and Emerging Adulthood

The transition from adolescence to adulthood is characterized by numerous challenges (Kessler \& Walters, 1998) and opportunities (Masten, Obradović, \& Burt, 2006) as young adults negotiate new adult roles and responsibilities (Schulenberg, Sameroff, \& Cicchetti, 2004). Relative to adolescents, emerging adults demonstrate more civic engagement, social competence, increased social capital and general life satisfaction (Eccles, Templeton, Barber, \& Stone, 2003; O'Connor et al., 2011) along with new perspectives on relationships and intimacy (Roisman, Masten, Coatsworth, \& Tellegen, 2004), personal identity (Schwartz, Klimstra, Luyckx, Hale III, \& Meeus, 2012), family obligations (Fuligni \& Pedersen, 2002) and work responsibilities (Roisman et al., 2004). These wide-ranging transitions suggest possible age-specificity in the epidemiology of risk-taking behavior, and specifically in the environmental impacts on those behaviors. For example, researchers have shown the importance of peer influences on risk taking 
behavior decreasing with age (Smith, Steinberg, Strang, \& Chein, 2015; Steinberg \& Monahan, 2007), including AOD use (Goldstick et al., 2017b).

AOD use places young people at risk for not completing developmental tasks of adolescence (Eaton et al., 2011) and early adulthood (Chassin, Flora, \& King, 2004) adding additional difficulty to the transition to adulthood. AOD-using youth may experience adjustment problems later in adolescence or in early adulthood (Bachman et al., 2002; Duncan, Alpert, Duncan, \& Hops, 1997). In particular, AOD use may inhibit development into healthy productive adults (Eaton et al., 2011), have a negative influence on intimate relationships (Van Ryzin, Fosco, \& Dishion, 2012), and lead to high rates of incarceration due to involvement in the drug trade (Jayakody, Danziger, \& Pollack, 2000), diminished job opportunities, and poverty (Aarons et al., 1999). Thus, the consequences of AOD use are likely to resonate across the period of emerging adulthood, but we know little about how, if at all, AOD use etiology varies across this important developmental transition. Researchers have shown both that a) the psychological effects of violence exposure depends on the timing of the exposure (Monahan, King, Shulman, Cauffman, \& Chassin, 2015); and that b) both AOD use (SAMSHA, 2013) and violence exposure (Loeber \& Farrington, 2012; Resnick et al., 2004; Stoddard et al., 2015; Sullivan, Farrell, \& Kliewer, 2006) change with age. Yet, age-specificity in the link between violence exposure and AOD use and how this agespecificity depends on the type of violence exposure has not been studied.

\section{Study Overview}

We used data from a longitudinal study of students at risk for high school drop-out in Flint, Michigan to characterize how the associations between three violence exposure variables (observed violence, violence victimization, and aggressive behavior) and past 30-day AOD use (marijuana and alcohol use) vary across the age range of roughly 14 to 23 . We applied varying-coefficient regression models (Hastie \& Tibshirani, 1993; S. Wood, 2006) - an extension of generalized linear models where the regression coefficient is a semi-parametric function of another variable - to these data to estimate how the regression coefficients relating violence exposure variables with the AOD (separately for alcohol and marijuana use) use outcomes vary, potentially non-linearly, as a function of age. Using this approach we will derive trajectories quantifying how the association between ETV and AOD use change across ages, which may suggest possible developmental components underlying that link.

\section{Methods}

Study Design and Setting

The data analyzed here comes from the Flint Adolescent Study (Zimmerman, Caldwell, \& Bernat, 2002), an ongoing longitudinal study of resiliency among urban residents that began in 1994. Eligible participants were youth attending one of four schools in Flint, Michigan and were considered at risk for 
high school drop-out (defined as an $8^{\text {th }}$ grade GPA of $\leq 3.0$ ). Individuals not identifying as Caucasian and/or African American, and those diagnosed with emotional and/or developmental impairs were not eligible for the study. During recruitment, $9799^{\text {th }}$ graders met the inclusion criteria; among those, $87 \%$ $(\mathrm{n}=850)$ participated in initial data collection. Those among the $13 \%$ that did not participate $\mathrm{n}=67$ were unable to be contacted after several attempts (e.g. school absences), $n=52$ no longer attended the public school, and $n=10$ refused. The initial cohort was $50 \%$ female, had an average age of 14.8 , and was $80 \%$ African American, 17\% Caucasian, and 3\% bi-racial (Caucasian/African American). The current study is restricted to the first 8 waves of data (roughly ages 14 to 23), and the analytic data set is restricted to those who had complete responses to substance use (past 30 day marijuana and alcohol use) questions and exposure to violence questions. In total, 5,362 observations met those criteria. Across waves, the average age ranged from 14.9 to 23.1 , percent female ranged from $50.2 \%$ to $57.7 \%$, and percent Black ranged from $79.5 \%$ to $82.2 \%$. All study procedures were approved by the University of Michigan IRB and all requirements for protection of human subjects were met.

Participants were assessed during roughly 60-minute interviews. Sensitive material was conveyed through paper and pencil questionnaires rather than verbally. The study includes three sets of four waves of data collected: high school (1994-1997), post-high school (1999-2002), and late twenties/early thirties (2008-2012). As the focus of this work is on adolescence and emerging adulthood, we only focus on the first two time periods here (waves 1-8). During that period, attrition rates were low, with an average of $90 \%$ successful follow-ups in the high school years, and $65 \%$ successful follow-ups in the post-high school years. In all analyses, only assessments with responses for every variable were used. Attrition Analysis

To justify the complete case analysis (i.e. use of only time points where all required measures were available), we conducted a brief attrition analysis. We examined baseline (i.e. wave 1) differences between individuals who were missing versus not missing at subsequent time points. In supplemental tables, we show 2-sample comparisons between those who were missing versus not missing at each of waves 2 through 8 for all study variables (Tables S1-S7). Overall, the average number of assessments per person was $6.30(\mathrm{SD}=1.77)$, and $91.4 \%$ of the 850 individuals were measured at no less than half of the waves. The only consistent correlate of missingness was sex, with males more likely to miss follow-ups than females at waves 3 through 8 . Among the 49 comparisons ( 7 variables at 7 waves) not involving sex, only two came out significant (violence perpetration at wave 2, and race at wave 5), indicating limited evidence (beyond sex differences) that those missing follow-ups were systematically different from those who did not. We conducted an adjusted analysis of attrition by using repeated measures logistic regression to find baseline correlates of missed follow-ups (1: present, 0: missed); those results are shown in Table S8. The adjusted analysis produced similar results to the unadjusted comparisons, with female 
VIOLENCE EXPOSURE AND AOD USE ACROSS AGES

gender corresponding to more than double the odds of being present at a given follow-up; no other variables were significant.

Measurements

Outcome variable. Separate analyses were conducted for both marijuana use and alcohol use as the outcome variables. Both measurements were taken on a 7-point scale measuring past 30-day frequency of use taken from Monitoring the Future study (Johnston, O'Malley, \& Bachman, 2002): 1(none), 2(1 to 2 times), 3(3 to 5 times), 4(6 to 9 times), 5(10 to 19 times), 6(20 to 39 times), and 7(more than 40 times).

Independent variables. We incorporated basic demographics (age, gender, race) into our descriptive analyses, but those were not included in the adjusted models, due to the inclusion of personlevel fixed effects as describe below (making the effects of time-invariant person-level covariates nonidentifiable). Our analysis focused primarily on the role of three scales capturing different aspects of past year exposure to violence: observed violence, violence victimization, and violence perpetration.

Observed violence was measured by the average response to two questions, asking how often in the past 12 months the respondent has: "Seen someone commit a violent crime where someone was hurt" and "Seen someone get shot, stabbed, or beaten up". Each question was measured on a scale from 1 (0 times) to 5 (4+ times). The two items comprising this scale had correlations between 0.64 and 0.75 across the waves, and 0.67 when aggregating all waves; expressed as Cronbach's $\alpha$, this corresponded to values ranging from 0.78 to 0.86 , with an aggregate total of 0.80 across waves.

Violence victimization was the average of three questions asking how often in the past 12 months the respondent has: "Had someone threaten you", "Had someone physically assault or hurt you", and "Had someone take something from you using physical force", each measured on a scale from 1 (0 times) to 5 (4+ times) and averaged. Cronbach's $\alpha$ ranged from 0.55 to 0.73 across waves, with $\alpha=0.63$ in aggregate.

Violence perpetration was measured by the average of five items capturing frequency of aggression and weapon carrying. Specifically, participants were asked how often in the past 12 months they had: "Intentionally hurt someone bad enough to need bandages or a doctor", "Used a knife or gun or some other thing to get something from a person", "Taken part in a fight where a group of your friends were against another group", "Carried a knife/razor", and "Carried a gun”. Each question was measured on a scale from 1 ( 0 times) to 5 (4+ times). Cronbach's $\alpha$ ranged from 0.60 to 0.78 across waves with $\alpha=$ 0.72 in aggregate.

In addition to ETV variables, we also included a measure of current friends' drug use, which was the average of five measurements quantifying on a five-point scale (1: None, 2: Some, 3: Many, 4: Most, 5: All) the perceived number of friends engaging in various drug use behaviors. The questions included 
the number who have a drug or alcohol problem, the number that use marijuana at least once a month, the number that have used cocaine, and the number that have been in legal trouble for selling drugs or using drugs (two separate questions). This scale has been found to be a strong correlate of substance use in prior studies using these data (Doljanec \& Zimmerman, 1998; Elkington, Bauermeister, \& Zimmerman, 2011; Goldstick et al, 2017b). This measure was included a time-varying exposure to guard against time-varying confounding in the models described below. Only this non-ETV time-varying exposure was selected a) for parsimony; and b) because peer behaviors are frequently observed as robust correlates of adolescent substance use (e.g. Goldstick et al, 2017b).

Statistical Analysis

We began with graphical and numerical descriptive statistics. We calculated descriptive statistics for basic demographics, the exposure to violence scales, friends' drug use, and marijuana/alcohol use scores at each wave. We also graphically displayed the unadjusted regression coefficient relating each exposure to violence scale with alcohol/marijuana use for each age from 14 to 23 by fitting separate linear regression models in each group to display how basic stratified analyses map onto the age-varying effects models fit subsequently.

Our primary analysis relied on generalized additive models (GAMs) (S. Wood, 2006), which are analogous to generalized linear models (GLMs) but relax the assumption that the predictors enter linearly in favor of a more general semi-parametric regression functions whose shape is empirically derived rather than pre-specified. In particular, we used GAMs to fit varying-coefficient regression models (Fan \& Zhang, 2008; Hastie \& Tibshirani, 1993). GAMs allow specification of regression models where the coefficient itself can be estimated as a potentially non-linear function of another variable (age in this analysis). The smoothness of the regression function (with linear being the smoothest possible) is determined within the fitting by a cross validation algorithm. When the variable that determines the varying coefficient is time, these are also known as time-varying effect models (Tan et al, 2012). Within this framework, the target of our inference is about whether the regression coefficient function, $\beta(a)$, is constant. Apart from allowing varying coefficients, the modeling framework used here is exactly analogous to a generalized linear model.

We initially use the GAM to estimate unadjusted smooth curve estimates of the age-varying regression coefficient for each ETV variable, separately for alcohol and marijuana use. These are used to display how the GAM output corresponds to the descriptive plots produced by the basic numerical summaries stratified by age. Next, we explore adjusted effects of each ETV variable using the GAM. We use individual-level fixed effects to adjust for any time-invariant confounding (Gunasekara, Richardson, Carter, \& Blakely, 2013), so that the resulting coefficient estimates are driven only by within-person (rather than between-person) effects. Because the person-level fixed effects capture all time-invariant 
individual-level covariate effects, we did not include factors like sex and race in those models. To account for potential time-varying confounding we also include perceived friends' drug use as a time-varying covariate in all models. Due to difficulties of fitting high-dimensional (in the parameters) varying coefficient models with multiple smooths in age simultaneously, we estimate the age-varying coefficient for each ETV measure one-at-a-time. In each model, the other ETV variables are included as timevarying covariates with fixed (non-age-varying) effects, to further guard against time-variant confounding. Within those models, we explicitly separate linear and non-linear interactions, as described in (S. Wood \& Wood, 2016), and test them separately to determine whether the more complex non-linear effects are necessary. The total within-person age-specific effects produced from that model, which are a combination of both the linear and non-linear interactions, are also displayed graphically. Although our outcome variables are unlikely to produce normally distributed residuals, their impact on the resulting inference is likely to be minimal given the sample size (Lumley, Diehr, Emerson, \& Chen, 2002), and thus we use the normal errors specification. All models are fit using the R package mgcv (S. Wood, 2006; S. N. Wood, 2012).

\section{Results}

Descriptive Statistics

Table 1 shows descriptive statistics on AOD use and ETV across the study waves. Levels of both marijuana and alcohol use generally increase across the waves. Marijuana use increased notably from Wave 1 to Wave 5, and was more stable afterwards. Alcohol use prior to Wave 6 (roughly when participants turned 21) was lower than marijuana use, but was higher thereafter. Rates of observed violence were highest in the first two waves and were lower thereafter. Rates of victimization and perpetration were highest in the first two waves and were at stable levels for Waves 3-8.

Figure 1 displays unadjusted regression coefficient estimates relating past 30 day marijuana and alcohol use with each of the four primary predictors, from age 14 to 23 , with the smooth age-varying regression coefficient estimate overlaid with $95 \%$ pointwise confidence intervals; ages 24 and 25 were omitted from the plot due to small sample sizes within those ages. While positive associations, and some trends are apparent (e.g. increasing relationship between violence perpetration and both alcohol and marijuana use) in the serial cross-sectional estimates, the large year-to-year fluctuation obscures the more precise understanding of the age trends that is shown in the smooth age-trajectories. Observed violence displays an increasing association with both alcohol and marijuana use; this association plateaus after about age 17 with regard to marijuana, and continues to increase linearly with regard to alcohol use. Violence victimization begins with a weak association between both alcohol and marijuana use, and those associations increase with age, with the rate of increase slowing down with regard to both substances; with regard to marijuana, the growth stops by age 18 , and by age 21 with alcohol. The associations 
between violence perpetration and both alcohol and marijuana use increase across the age span under study, showing linear growth with regard to alcohol use, and mostly linear growth with regard to marijuana, with some slowing in the growth rate at roughly ages 16-18 with regard to marijuana use. Adjusted within-person models

Results from the adjusted models targeting within-person associations between violence exposure and AOD use are shown in Table 2 and Figure 2. Beginning with marijuana use, the age-invariant model indicates strong risk-enhancing effects of friends' drug use, observed violence, and violence perpetration on levels of marijuana use. Higher violence victimization rates correspond to lower of levels of marijuana use. There was evidence of non-linear age-dependence in the within-person association between marijuana use and all three violence exposure variables studied here. The positive association between observed violence and marijuana use increased until about age 18, where it peaked and decreased thereafter; overall, based on the pointwise confidence intervals being entirely above zero, observed violence corresponded to higher levels of marijuana use from roughly ages 16-22 (Figure 2). Violence victimization was most negatively associated with marijuana use at younger ages and corresponded to a positive association thereafter, becoming non-significant by age 21 (Figure 2). Violence perpetration was increasingly associated with violence from ages 14-17, plateauing to a large age-invariant association at about age 17 (Figure 2).

There was evidence of age-specific within-person associations between alcohol use and both observed violence and violence perpetration (Table 2). There was no evidence of a linear or non-linear age-dependence in the association between alcohol use and victimization (Table 2). The association between observed violence and alcohol use increased linearly with age, in contrast with marijuana use (Figure 2), showing a nearly 4-fold larger effect than on alcohol than marijuana use by age 23. Violence perpetration and alcohol use showed an increasing association over time, with the growth rate of the stopping by around age 21 (Figure 2). The pointwise confidence intervals for the effect of victimization on alcohol use covered zero across over most of the trajectory (Figure 2).

\section{Discussion}

By studying the relationship between past 30-day AOD use (marijuana/alcohol) and three measures related to violence exposure - observed violence, violence perpetration, and violence victimization - across adolescence and young adulthood, we found evidence that the association between AOD use and violence exposure generally increases with age. Contrasting our results with simple visual analysis of effect estimates across arbitrary age groups emphasizes the utility of borrowing information across ages to estimate a smooth effect trajectory that is ultimately more interpretable. Overall, violence perpetration showed the largest within-person associations with both types of substance use. The association between marijuana use and violence perpetration increased until middle adolescence ( age 
17); this increase continued longer into development ( age 21) for alcohol use. In other words, violence perpetration and AOD use are more closely linked in the later stages of adolescence/early adulthood, and those age-specific increases continued later with regard to alcohol use. The association between observed violence and substance use was qualitatively similar but smaller in magnitude; the positive association between alcohol use and observed violence continued to increase across the entire age range studied here, while the association between marijuana use and observed violence peaked around age 18, showing some evidence of decreasing thereafter. While unadjusted analyses indicated that violence victimization became increasingly risk-enhancing with age, our within-person analysis suggests that those findings were driven more by between-person differences. Across most of the age range studied here, changes in violence victimization did not correspond to changes in AOD use within-person, and at the youngest ages (14-15) there was evidence of a negative association. Although the observational nature of the data and the measurements employed prohibit a causal interpretation or elucidation of the mechanisms at work, several explanations for our results are possible.

One general explanation that applies to both substances relates to problem behavior theory, which suggests that problem behaviors often cluster due to their similar roles in signifying rebellion or common psychological distresses (Jessor, 1991). Few researchers, however, have studied how problem behaviors cluster differentially as a function of age. Our results indicate that within-person changes in violence perpetration correlate more strongly with changes in substance use behavior at older ages than younger ages. Rates of aggressive behavior generally decrease after adolescence (Perkins, 1997), but our results indicate that those whose rates of violence perpetration increase at later ages are more likely to have concurrent increases in AOD use behavior. In other words, aggressive behavior and AOD may cluster together more closely at older ages than at younger ages. More generally, our findings could be explained by the diverse set of stressors adolescents are exposed to which may also correlate with AOD use, thus muddying the precise AOD use-violence exposure relationships. In other words, as an individual ages, adolescence-specific stressors may dissipate, allowing exposure to violence to emerge as a strong correlate of AOD use at later ages, reflecting a differential clustering of behaviors. This explanation is consistent with the observed age-variation in the association between violence perpetration and AOD use, and the lack of analogous findings with regard to violence victimization.

Changes in the relative magnitude of the effects of violence exposure on marijuana, and alcohol use, levels, respectively, could indicate that substance availability plays a role in the observed agevariation. Specifically, given that marijuana was completely illegal at the time (1995-2002, when even medicinal marijuana was not legal in Michigan), marijuana availability was less age-dependent, while alcohol becomes increasingly available with age, and individuals and their friends reach legal drinking age. Even after medical marijuana became available, it required a much more complicated process (e.g., 
health insurance, doctor appointment and approval, specific locations for pickup) than purchasing alcohol at any of a number of outlets with only an ID. This supposition is borne out by the descriptive statistics indicating that marijuana use levels were level after Wave 3, while alcohol use rates increased notably after Wave 6 (when many participants turned 21), which is also consistent with nationally representative data indicating past year marijuana use rates plateau in early adulthood ( age 18$)$, while drinking rates continue to increase into the early-to-mid 20s (SAMSHA, 2013). Our findings could indicate that, at younger ages, marijuana was more available (while equally unlawful), and perhaps easier to discretely store and transport than alcohol, and thus was used more frequently in response to violence exposure. This, combined with alcohol becoming the more frequently used substance in later waves, supports the idea that availability plays a role in our results.

Another possible explanation of our results is that some findings may arise from changes in the settings where AOD use takes place, or the circumstances surrounding its procurement, among youth. Consistent with prior literature (e.g. Goldstick et al., 2015), we did find a strong association between substance use and aggressive behavior, adding a further nuance that this association generally increases with age. In addition to alcohol use being less frequent among youth (due to lowered availability), the illegality of alcohol for those under age 21 makes its use more likely to occur in non-public settings, rather than public settings like bars. Thus, underage drinkers may have less exposure to the interpersonal contact that can facilitate alcohol-related physical aggression. The disinhibitory effect of alcohol, which can correspond to violent behavior, particularly in those predisposed to aggression (Giancola, 2002), is likely to be at least partially immutable over the age range studied here. Thus, the association between alcohol use and violence perpetration, which increases through adolescence then plateaus at approximately age 21 , is consistent with the idea that increasing alcohol availability, and the ability to drink in public, increases opportunities for alcohol-related aggression. This is consistent with prior literature indicating that public drinking contexts (as opposed to drinking in private) are more associated with drinking-related violence (Wells et al, 2005). In contrast, the association between marijuana use and violence perpetration increased from age 14-16, and plateaued thereafter. The illegality of marijuana during this study period means it necessitates contact with the illegal drug trade (Friedman, Glassman, \& Terras, 2001) which may be associated with violence perpetration. Increases in that exposure in early adolescence (due to increased use rates seen in waves 1-3), but not in adulthood, may explain the age dynamic results seen. Whatever the explanation, our overall findings suggest that AOD use interventions focused on those exposed to violence as observer or aggressor (or both), may be most productive among older individuals.

Interestingly, we did not find within-person variations in violence victimization to be associated with variations in AOD use at most ages. The contrast of that finding with the descriptive analyses, which 
showed notable associations between victimization and AOD use, indicates that much of that association was due to between-person differences. That is, across ages, those with low violence exposure had lower rates of AOD use, but we found little evidence that variations in victimization corresponded to AOD use variations within an individual. While the within-person effects for violence perpetration and observed violence were smaller than the overall, unadjusted, effects, they still remained non-negligible - and qualitatively similar - unlike the findings for victimization. In fact, at younger ages, victimization was associated with less marijuana use. It is possible that some of the violence exposure occurred during the procurement of marijuana resulting is less use (and therefore less interaction with the unlawful and perhaps more dangerous drug trade) as a way to reduce exposure.

We now note some limitations of this work. First, our primary exposures and outcomes are based on self-report data. While individuals were assured confidentiality, their reporting of their own AOD use behavior or their violence exposure is subject to error. Yet, researchers have noted in bogus pipeline studies that self-reported AOD use is mostly accurate, and to guard against potential response bias introduced in an interview context these questions were included in a paper and pencil format at the end of the interview portion of the data collection. In addition, individuals' perceptions about their violence exposure are perhaps more relevant than any more objective measure of exposures may reflect. Second, our study population is predominantly African American and from a single city. Given the demographic similarities between Flint and other small economically challenged urban contexts (e.g., Youngstown, $\mathrm{OH}$; Camden, NJ), our results may generalize beyond Flint. In addition, given the significant health disparities facing predominantly African American populations in such disadvantaged cities (CDC, 2005), this study population is important to study in its own right. Third, the latter part of the time period studied had some attrition $(\sim 35 \%)$. Our attrition analysis indicated that only gender was predictive of attrition risk and that the primary variables studied here (AOD use, ETV) did not correspond to differential risk for attrition. In addition, we note that $89 \%$ of the baseline cohort was measured in at least one time point during the latter half of the study, indicating the effects of non-response bias on our final results is likely to be minimal. Fourth, the GPA cut-off of 3.0 is not a high-specificity threshold for dropout risk. Thus, many of the individuals recruited for the study may not have been at particularly high risk for drop-out, but near all individuals at high-risk for drop-out would meet that eligibility criteria (i.e. it is a highsensitivity threshold). In light of this, our study population may be best viewed as a general sample of high-school youth in Flint, excluding those with very low drop-out risk. Finally, our data is limited by its failure to capture cumulative violence exposure. While summation of ETV variables to measure cumulative exposure is possible, this would bias the cumulative violence measure to those with more follow-ups measured, which would present a larger limitation than the failure to measure cumulative 
violence exposure, particularly given that the focus of this study was on temporal co-occurrence of AOD use and ETV, rather than the effect of cumulative exposure.

Conclusions

Alleviating the health disparities in the United States requires understanding the effects of one of its key correlates: violence exposure. This study has elucidated how the association between violence exposure and AOD use varies as a function of age. In particular, we found the association between AOD use and violence exposure increases with age and that some of these changes may correspond to agespecific changes in substance availability, handling of the stress incurred by violence exposure locales where substances tend to be used, or age-specific behavioral clustering; further studies are required to elucidate the mechanism for the age-specific differences identified here. Our findings indicate higher ETV/AOD comorbidity among older emerging adults, and the implications are two-fold: a) programs addressing both AOD use and violence concurrently, which have been shown to be effective (e.g. Walton et al, 2010), may be most effective among that age group; and b) prevention programs focusing on adolescents and emerging adults exposed to violence should include content related to AOD use, particularly among older emerging adults. Future studies that include contemporaneous measurements of drinking contexts, stress and coping behaviors, and substance use motives could help to determine the plausibility of the competing explanations for our results discussed here. Those potential findings could inform age-specific substance use prevention programs by determining the most salient content for particular developmental stages.

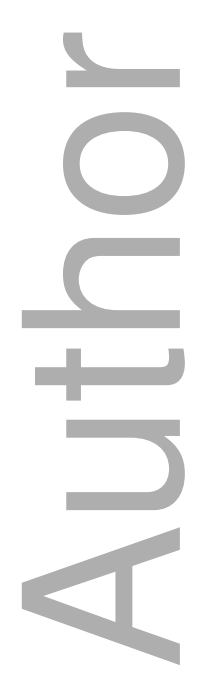


Figure 1: Association between ETV variables (left to right: observed violence, violence victimization, violence perpetration) and past 30-day marijuana use (top) and alcohol use (bottom) in ages 14 to 23 . Solid lines represent cross-sectional regression coefficient estimates (with age rounded to the nearest year) and dashed lines reflect smooth age-varying regression coefficient estimates of the same data.
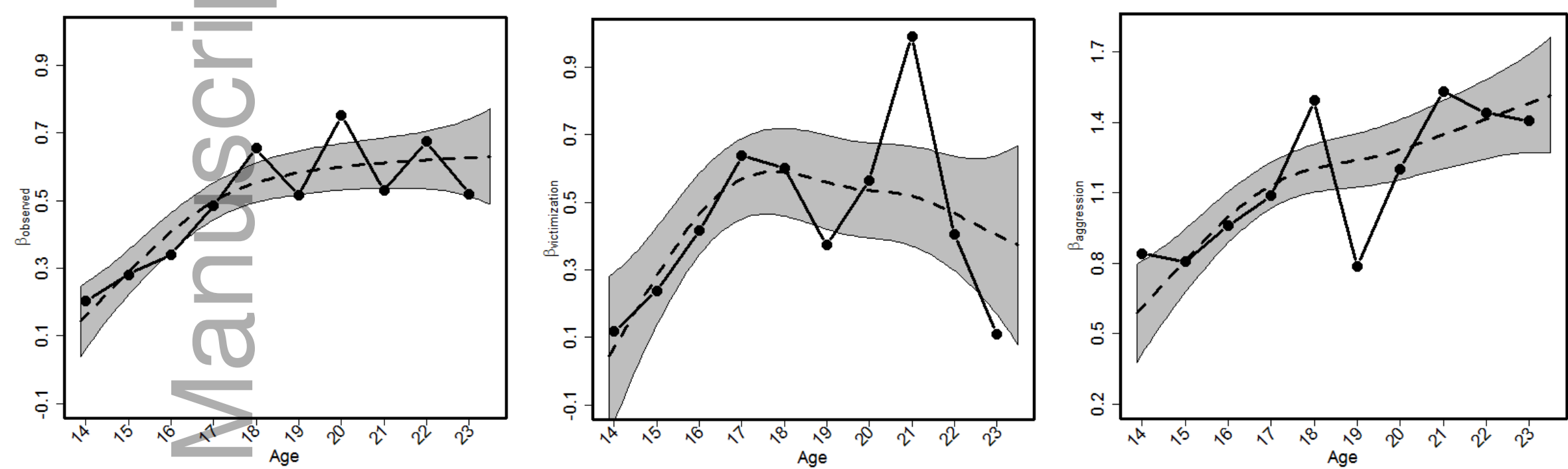

This article is protected by copyright. All rights reserved 

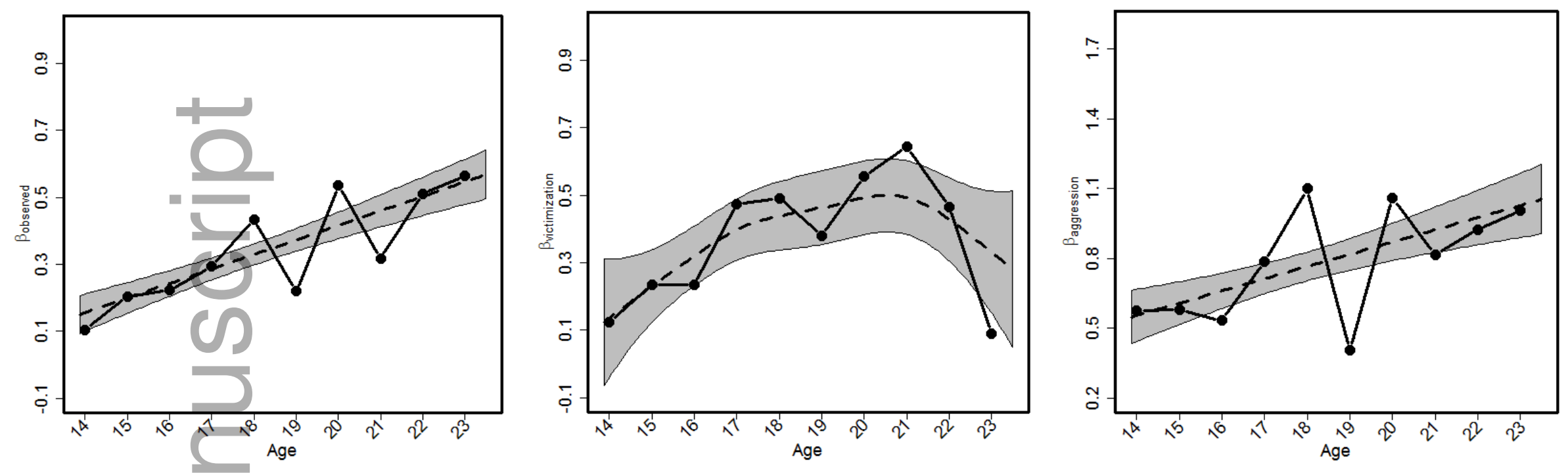

Table 1. Descriptive statistics for the analytic sample composed of every assessment where each substance use measurements and violence exposure measurement was available. Unless specified as a percentage, entries are mean (SD)

\begin{tabular}{|c|c|c|c|c|c|c|c|c|c|}
\hline & Wave 1 & Wave 2 & Wave 3 & Wave 4 & Wave 5 & Wave 6 & Wave 7 & Wave 8 & Total \\
\hline Total number assessed $\dagger$ & 850 & 812 & 783 & 770 & 572 & 639 & 576 & 579 & 5581 \\
\hline $\begin{array}{l}\text { Total number analyzable } \dagger \\
\text { Individual factors }\end{array}$ & 847 & 775 & 739 & 725 & 546 & 626 & 537 & 567 & 5362 \\
\hline Female $(\%)$ & $50.2 \%$ & $51.1 \%$ & $51.7 \%$ & $52.3 \%$ & $57.7 \%$ & $54.2 \%$ & $55.5 \%$ & $55.0 \%$ & $53.1 \%$ \\
\hline African American (\%) & $80.3 \%$ & $79.5 \%$ & $79.6 \%$ & $79.7 \%$ & $82.2 \%$ & $80.2 \%$ & $79.9 \%$ & $81.3 \%$ & $80.2 \%$ \\
\hline Age & $14.9(0.6)$ & $15.9(0.6)$ & $16.8(0.6)$ & $17.8(0.6)$ & $20.0(0.6)$ & $21.0(0.6)$ & $22.1(0.6)$ & $23.1(0.7)$ & $18.5(2.9)$ \\
\hline Friends' drug use $\dagger \dagger$ & $1.3(0.4)$ & $1.5(0.4)$ & $1.7(0.7)$ & $1.7(0.7)$ & $1.7(0.8)$ & $1.7(0.8)$ & $1.8(0.8)$ & $1.7(0.8)$ & $1.6(0.7)$ \\
\hline
\end{tabular}

This article is protected by copyright. All rights reserved 
Substance Use

\begin{tabular}{|c|c|c|c|c|c|c|c|c|c|}
\hline Marijuana use & $1.6(1.3)$ & $1.9(1.6)$ & $1.9(1.7)$ & $2.1(1.9)$ & $2.1(2.0)$ & $2.0(1.9)$ & $2.2(2.0)$ & $2.2(2.1)$ & $2.0(1.8)$ \\
\hline Alcohol use & $1.5(1.0)$ & $1.6(1.0)$ & $1.6(1.2)$ & $1.7(1.2)$ & $2.0(1.3)$ & $2.1(1.5)$ & $2.4(1.6)$ & $2.3(1.6)$ & 18 \\
\hline
\end{tabular}

Exposure to Violence

Observed Violence

$2.2(1.2) \quad 2.2(1.2) \quad 1.9(1.1) \quad 1.7(1.0) \quad 1.6(1.0) \quad 1.6(1.0) \quad 1.5(0.9) \quad 1.6(1.0) \quad 1.8(1.1)$

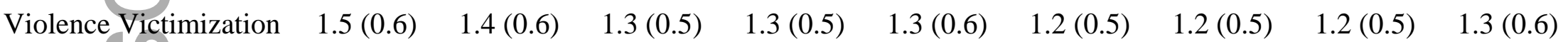

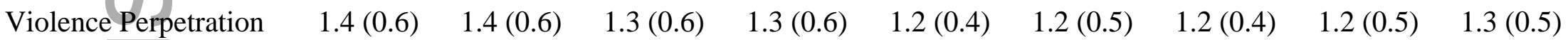

$\dagger$ : Total number assessed are the number who were successfully followed up for the study. Number analyzable corresponded to those with complete substance use and exposure to violence measurements.

$\dagger \dagger$ : In waves 1-8, there were $22,16,7,4,24,11,24$, and 17 individuals missing the friends' drug use variable, respectively. In all subsequent unadjusted analyses, those individuals were included. In the adjusted analysis, they were not.

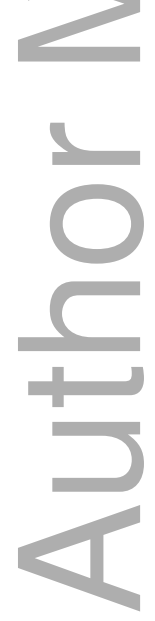

This article is protected by copyright. All rights reserved 
Table 2: Age-varying coefficient regression model stratified by age, and unstratified.

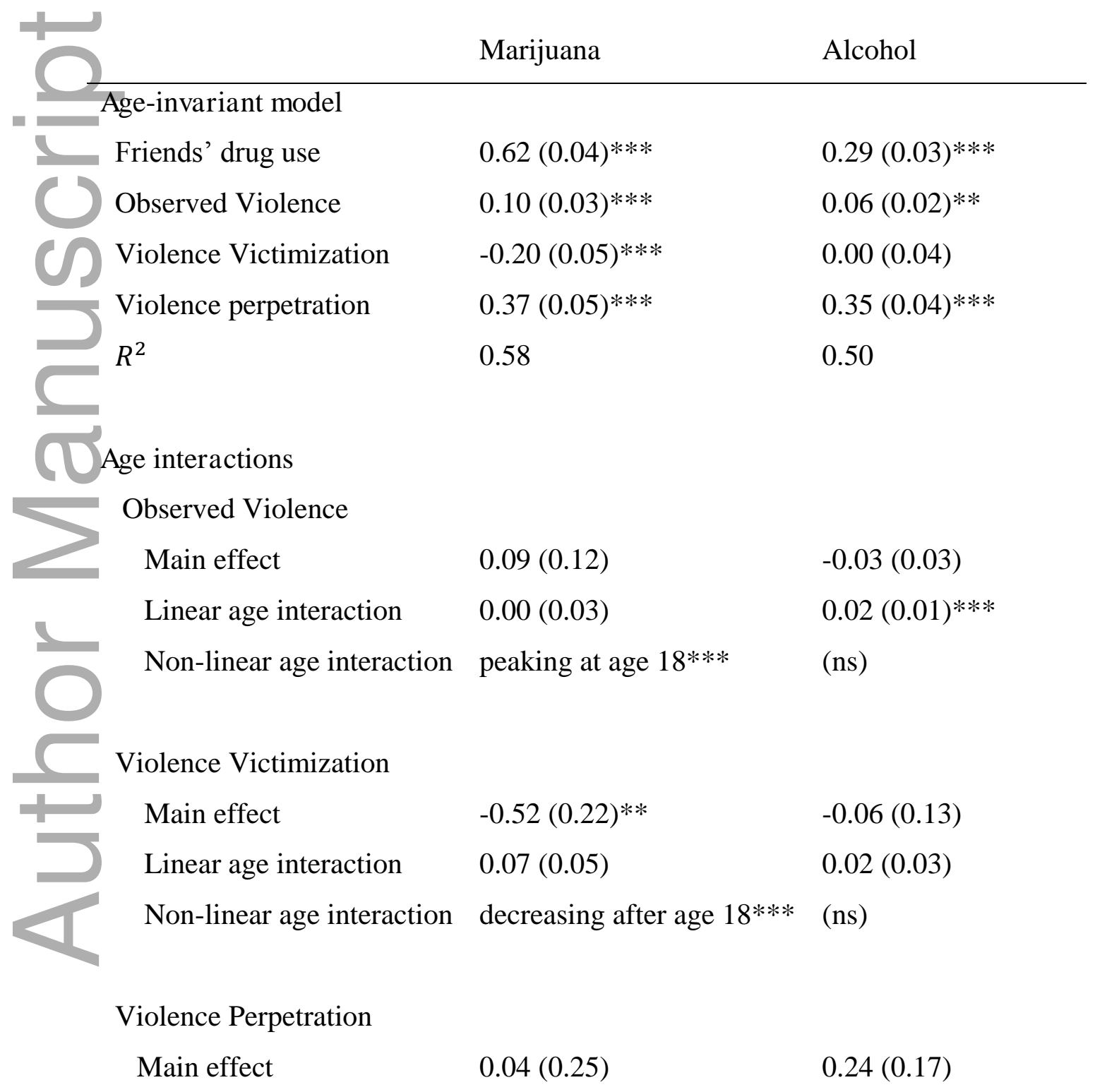

This article is protected by copyright. All rights reserved 


\section{Linear age interaction $\quad 0.07(0.05)$}

Non-linear age interaction
$0.03(0.04)$

plateauing at age $18^{*}$

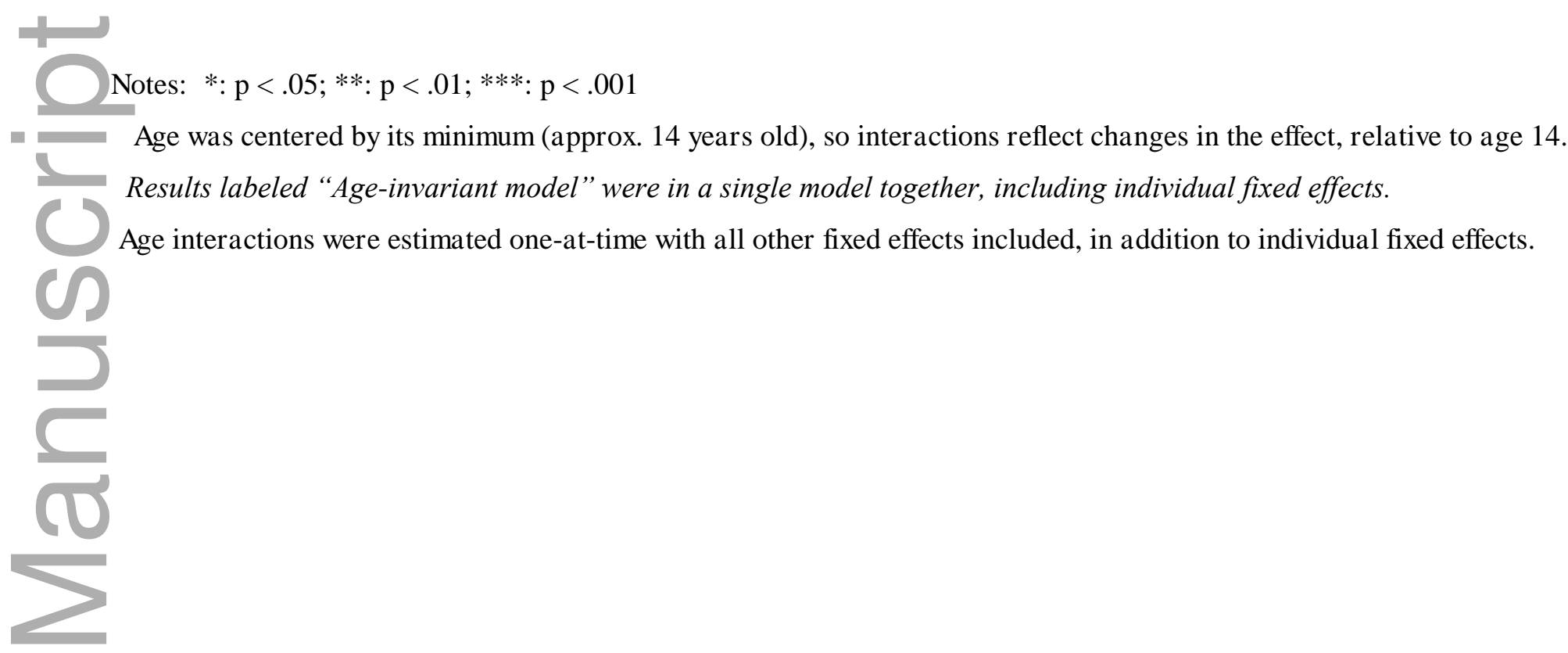

Figure 2: Adjusted smooth-age varying ETV (left: observed; middle: victimization; right: aggression/perpetration) effects on past 30 day marijuana (top) and alcohol (bottom) use; time-invariant individual-level fixed effects, and time-varying covariates (all other violence exposures, and friends' drug use) was controlled for so that covariate effect trajectories reflect within-individual effects as a function of age.

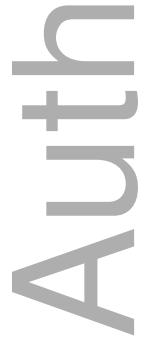

This article is protected by copyright. All rights reserved 
VIOLENCE EXPOSURE AND AOD USE ACROSS AGES
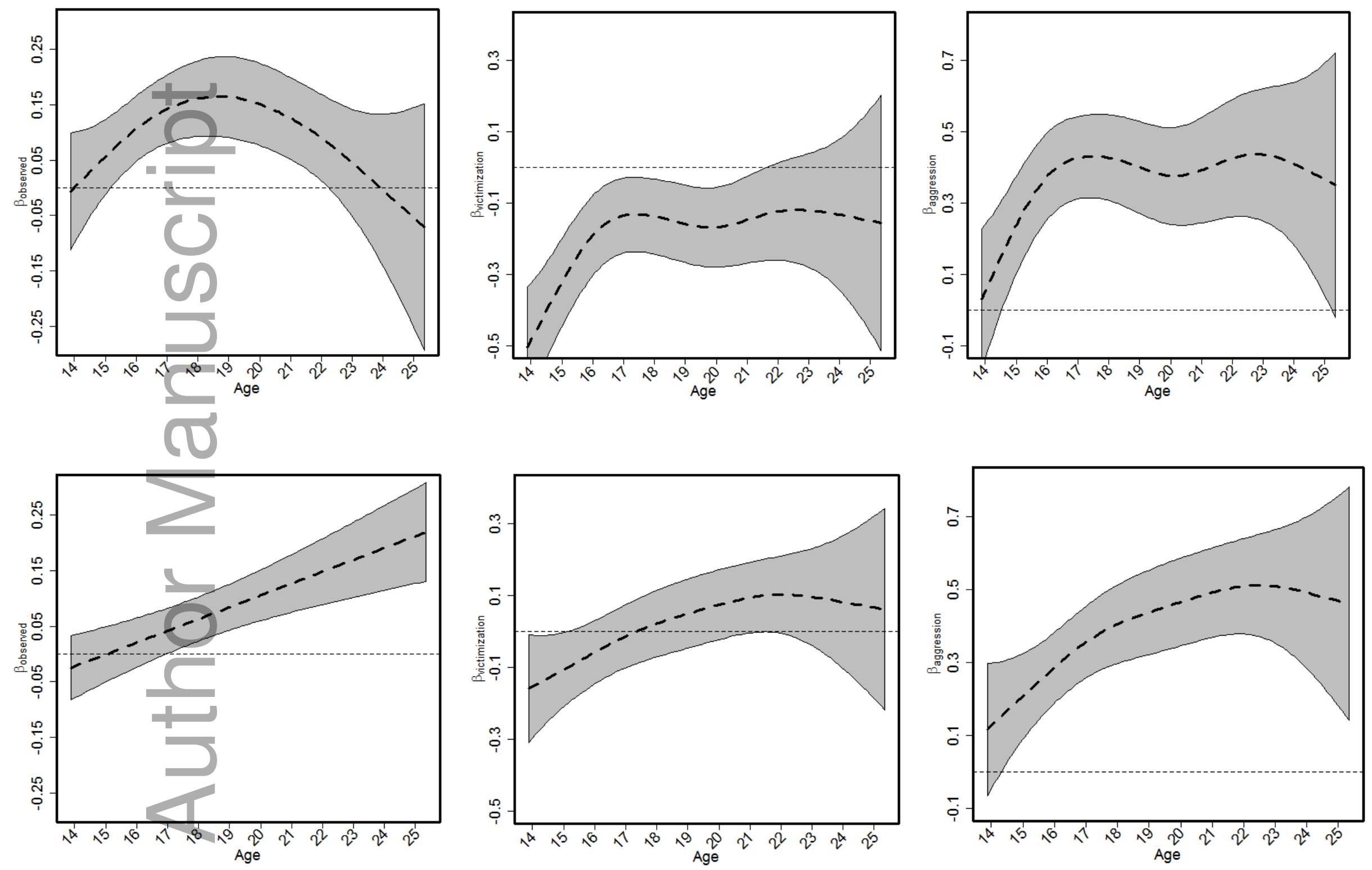

This article is protected by copyright. All rights reserved 
VIOLENCE EXPOSURE AND AOD USE ACROSS AGES

\section{$\underline{\text { References }}$}

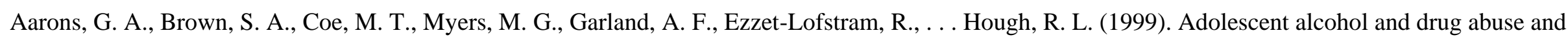
health. Journal of Adolescent Health, 24(6), 412-421.

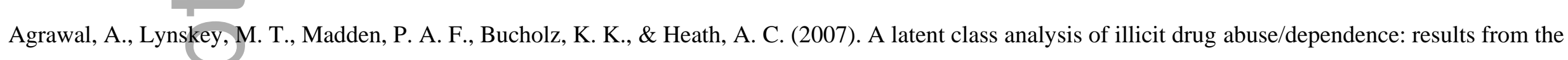
National Epidemiological Survey on Alcohol and Related Conditions. Addiction, 102(1), 94-104.

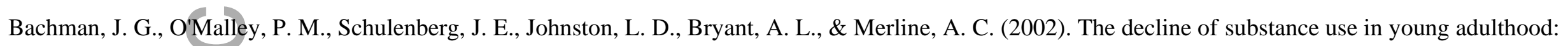
Changes in social activities, roles, and beliefs.

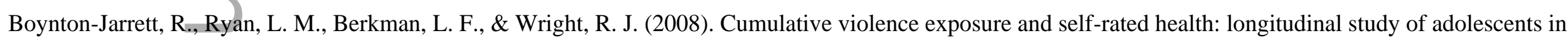
the United States. Pediatrics, 122(5), 961-970.

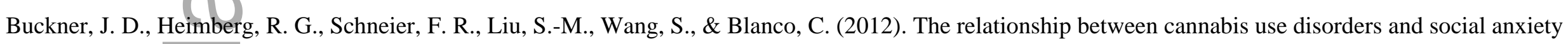
disorder in the National Epidemiological Study of Alcohol and Related Conditions (NESARC). Drug and Alcohol Dependence, 124(1), 128-134.

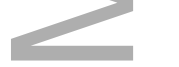

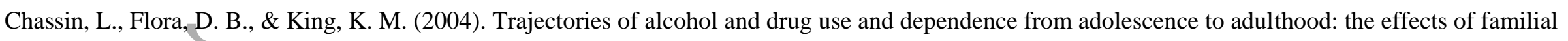
alcoholism and personality. Journal of abnormal psychology, 113(4), 483.

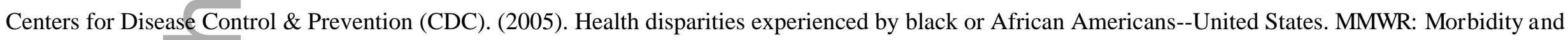
mortality weekly report, 54(1), 1-3.

Dahlberg, L. L., \& Potter, L. B. (2001). Youth violence: Developmental pathways and prevention challenges. Am J Prev Med, $20(1)$, 3-14.

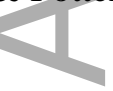

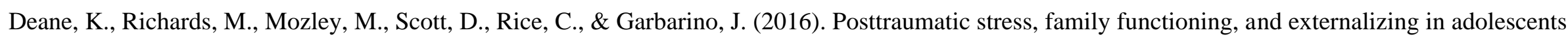
exposed to violence: a moderated mediation model. Journal of Clinical Child \& Adolescent Psychology, 1-14.

This article is protected by copyright. All rights reserved 
Duncan, S. C., Alpert, A., Duncan, T. E., \& Hops, H. (1997). Adolescent alcohol use development and young adult outcomes. Drug and Alcohol Dependence, 49(1), 39-48.

Eaton, D. K., Kann, L., Kinchen, S., Shanklin, S., Ross, J., Hawkins, J., . . Chyen, D. (2011). Youth risk behavior surveillance-United States, 2009. Morbidity and Mortality Weekly Report, 59.

-

Eccles, J., Templeton, J., Barber, B., \& Stone, M. (2003). Adolescence and emerging adulthood: The critical passage ways to adulthood.

Elkington, K. S., Bauermeister, J. A., \& Zimmerman, M. A. (2011). Do parents and peers matter? A prospective socio-ecological examination of substance use and sexual risk among African American youth. Journal of adolescence, 34(5), 1035-1047.

Epstein-Ngo, Q. M., Cunningham, R. M., Whiteside, L. K., Chermack, S. T., Booth, B. M., Zimmerman, M. A., \& Walton, M. A. (2013). A daily calendar analysis of substance use and dating violence among high risk urban youth. Drug and alcohol dependence, 130(1), 194-200.

Epstein-Ngo, Q. M., Walton, M. A., Chermack, S. T., Blow, F. C., Zimmerman, M. A., \& Cunningham, R. M. (2014). Event-level analysis of antecedents for youth violence: Comparison of dating violence with non-dating violence. Addictive behaviors, 39(1), 350-353.

Fagan, A. A., Wright, E. M., \& Pinchevsky, G. M. (2015). Exposure to violence, substance use, and neighborhood context. Social Science Research, 49, $314-326$. doi: http://dx.doi.org/10.1016/j.ssresearch.2014.08.015

Fan, J., \& Zhang, W. (2008). Statistical methods with varying coefficient models. Statistics and its Interface, 1(1), 179.

Faraway, J. J. Linear models with R. 2005. Boca Raton, Florida: Chapman\&Hall/CRC, Google Scholar.

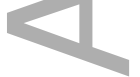

Finkelhor, D., Ormrod, R., Turner, H., \& Hamby, S. L. (2005). The victimization of children and youth: A comprehensive, national survey. Child maltreatment, $10(1), 5-25$.

This article is protected by copyright. All rights reserved 
Friedman, A. S., Glassman, K., \& Terras, A. (2001). Violent behavior as related to use of marijuana and other drugs. Journal of Addictive Diseases, 20 (1), 49-72.

Fuligni, A. J., \& Pedersen, S. (2002). Family obligation and the transition to young adulthood. Dev Psychol, 38(5), 856.

Fuller-Thomson, E., Roane, J. L., \& Brennenstuhl, S. (2016). Three Types of Adverse Childhood Experiences, and Alcohol and Drug Dependence Among Adults: An Investigation Using Population-Based Data. Subst Use Misuse, 51(11), 1451-1461. doi: 10.1080/10826084.2016.1181089

a

Giancola, P. R. (2002). The influence of trait anger on the alcohol-aggression relation in men and women. Alcoholism: Clinical and Experimental Research, 26(9), $1350-1358$

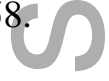

Goldstick, J. E., Carter,P. M., Walton, M. A., Dahlberg, L. L., Sumner, S. A., Zimmerman, M. A., \& Cunningham, R. M. (2017a). Development of the SaFETy Score: A Clinical Screening Tool for Predicting Future Firearm Violence Risk. Annals of internal medicine, 166(10), $707-714$.

Goldstick, J. E., Heinze, J., Ngo, Q., Hsieh, H.-F., Walton, M. A., Cunningham, R. M., \& Zimmerman, M. A. (2017b). Perceived Peer Behavior and Parental Support as Correlates of Marijuana Use: The Role of Age and Gender. Substance Use and Misuse. 1-11 (Published online).

Goldstick, J. E., Lipton, R. I., Carter, P., Stoddard, S. A., Newton, M. F., Reischl, T., . . Cunningham, R. M. (2015). The effect of neighborhood context on the relationship between substance misuse and weapons aggression in urban adolescents seeking ED care. Substance use \& misuse, 50(5), 674-684.

Gorman-Smith, D., Henry, D. B., \& Tolan, P. H. (2004). Exposure to community violence and violence perpetration: The protective effects of family functioning. Journal of Clinical Child and Adolescent Psychology, 33(3), 439-449.$$
\text { (1) }
$$

Gunasekara, F. I., Richardson, K., Carter, K., \& Blakely, T. (2013). Fixed effects analysis of repeated measures data. International journal of epidemiology, 43(1), 264-269.

Hastie, T., \& Tibshirani, R. (1993). Varying-coefficient models. Journal of the Royal Statistical Society. Series B (Methodological), 757-796.

This article is protected by copyright. All rights reserved 
Herrenkohl, T. I., Maguin, E., Hill, K. G., Hawkins, J. D., Abbott, R. D., \& Catalano, R. F. (2000). Developmental risk factors for youth violence. J Adolesc Health, 26(3), 176-186.

Jayakody, R., Danziger, S., \& Pollack, H. (2000). Welfare reform, substance use, and mental health. Journal of Health Politics, Policy and Law, $25(4), 623-652$.

Jessor, R. (1991). Risk behavior in adolescence: A psychosocial framework for understanding and action. Journal of adolescent Health, 12(8), 597-605.

Johnston, L. D., O'Malley, P. M., \& Bachman, J. G. (2002). MONITORING THE FUTURE NATIONAL RESULTS ON ADOLESCENT DRUG USE Overview of Key Findings. from http://www.monitoringthefuture.org/pubs/monographs/overview2002.pdf.

Kessler, R. C., \& Walters, E. E. (1998). Epidemiology of DSM-III-R major depression and minor depression among adolescents and young adults in the national comorbidity survey. Depression and anxiety, 7(1), 3-14.

Kitzmann, K. M., Gaylord, N. K., Holt, A. R., \& Kenny, E. D. (2003). Child witnesses to domestic violence: a meta-analytic review. Journal of consulting and clinical psychology, 71(2), 339.

Loeber, R., \& Farrington, D. P. (2012). From juvenile delinquency to adult crime: Criminal careers, justice policy and prevention: Oxford University Press.

Lumley, T., Diehr, P., Emerson, S., \& Chen, L. (2002). The importance of the normality assumption in large public health data sets. Annual review of public health, 23(1), 151-169.

Masten, A. S., Obradović, J., \& Burt, K. B. (2006). Resilience in Emerging Adulthood: Developmental Perspectives on Continuity and Transformation.

McGovern, M. P., Lambert-Harris, C., Acquilano, S., Xie, H., Alterman, A. I., \& Weiss, R. D. (2009). A cognitive behavioral therapy for co-occurring substance use and posttraumatic stress disorders. Addictive behaviors, 34(10), 892-897. doi: $\underline{\text { http://dx.doi.org/10.1016/j.addbeh.2009.03.009 }}$

This article is protected by copyright. All rights reserved 
VIOLENCE EXPOSURE AND AOD USE ACROSS AGES

Monahan, K. C., King, K. M., Shulman, E. P., Cauffman, E., \& Chassin, L. (2015). The effects of violence exposure on the development of impulse control and future orientation across adolescence and early adulthood: Time-specific and generalized effects in a sample of juvenile offenders. Dev Psychopathol, 27(4 Pt 1), 1267-1283. doi: 10.1017/s0954579414001394

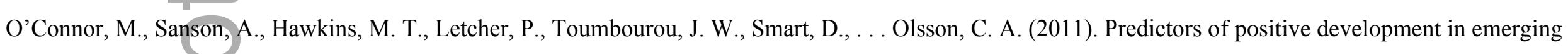
adulthood. J Youth Adolesc, 40(7), 860-874.

(

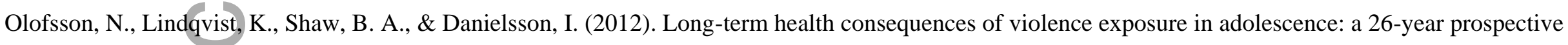
study. BMC Public Health, 12, 411. doi: 10.1186/1471-2458-12-411

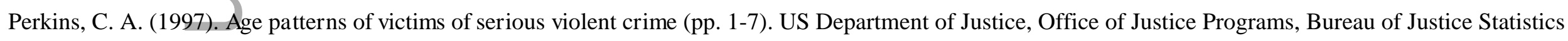

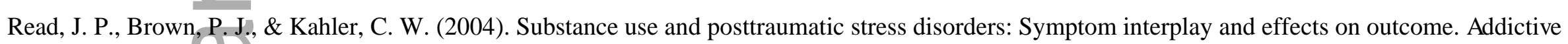
behaviors, 29(8), 1665-1672.

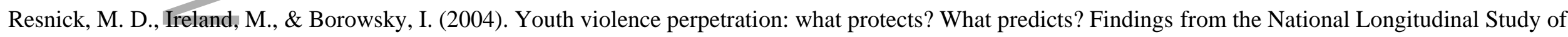
Adolescent Health. Journal of Adolescent Health, 35(5), 424. e421-424. e410.

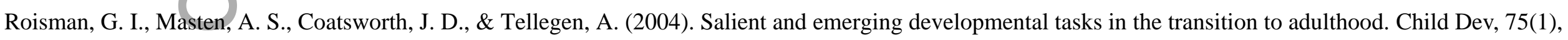
123-133.

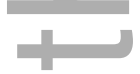

SAMSHA. (2013). Results from the 2013 National Survey on Drug Use and Health: Detailed Tables. Retrieved 9/15/2017 from http://archive.samhsa.gov/data/NSDUH/2013SummNatFindDetTables/DetTabs/NSDUH-DetTabsSect1peTabs1to46-2013.htm\#tab1.12b

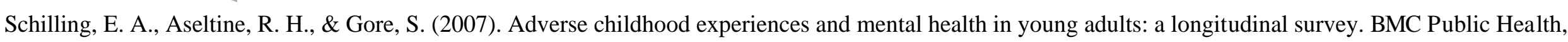
$7(1), 1$.

This article is protected by copyright. All rights reserved 
VIOLENCE EXPOSURE AND AOD USE ACROSS AGES

Schulenberg, J. E., Sameroff, A. J., \& Cicchetti, D. (2004). The transition to adulthood as a critical juncture in the course of psychopathology and mental health.

Development and psychopathology, 16(04), 799-806.

Schwartz, S. J., Klimstra, T. A., Luyckx, K., Hale III, W. W., \& Meeus, W. H. (2012). Characterizing the self-system over time in adolescence: Internal structure and associations with internalizing symptoms. J Youth Adolesc, 41(9), 1208-1225.

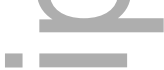

Sheidow, A. J., Henry, D. B., Tolan, P. H., \& Strachan, M. K. (2014). The role of stress exposure and family functioning in internalizing outcomes of urban families. Journal of child and family studies, 23(8), 1351-1365.

Smith, A. R., Steinberg, L., Strang, N., \& Chein, J. (2015). Age differences in the impact of peers on adolescents' and adults' neural response to reward. Developmental cognitive neuroscience, 11, 75-82.

Springer, K. W., Sheridān, J., Kuo, D., \& Carnes, M. (2007). Long-term physical and mental health consequences of childhood physical abuse: results from a large population-based sample of men and women. Child Abuse Negl, 31(5), 517-530. doi: 10.1016/j.chiabu.2007.01.003

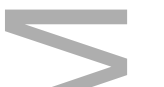

Steinberg, L., \& Monahan, K. C. (2007). Age differences in resistance to peer influence. Developmental psychology, $43(6), 1531$.

Stoddard, S. A., Epstein-Ngo, Q., Walton, M. A., Zimmerman, M. A., Chermack, S. T., Blow, F. C., . . Cunningham, R. M. (2015). Substance use and violence among youth: a daily calendar analysis. Subst Use Misuse, 50(3), 328-339. doi: 10.3109/10826084.2014.980953

Sullivan, T. N., Farrell, A. D., \& Kliewer, W. (2006). Peer victimization in early adolescence: association between physical and relational victimization and drug use, aggression, and delinquent behaviors among urban middle school students. Dev Psychopathol, 18(1), 119-137. doi: 10.1017/S095457940606007X

Turner, H. A., Shattuck, A., Finkelhor, D., \& Hamby, S. (2016). Polyvictimization and youth violence exposure across contexts. Journal of Adolescent Health, 58(2), 208-214.

This article is protected by copyright. All rights reserved 
Van Ryzin, M. J., Fosco, G. M., \& Dishion, T. J. (2012). Family and peer predictors of substance use from early adolescence to early adulthood: An 11-year prospective analysis. Addictive behaviors, 37(12), 1314-1324.

Vermeiren, R., Schwab-Stone, M., Deboutte, D., Leckman, P. E., \& Ruchkin, V. (2003). Violence exposure and substance use in adolescents: findings from three countries. Pediatrics, 111(3), 535-540.

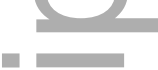

Walton, M.A., Chermack, S.T., Shope, J.T., Bingham, C.R., Zimmerman, M.A., Blow, F.C. and Cunningham, R.M. (2010). Effects of a brief intervention for reducing violence and alcohol misuse among adolescents: a randomized controlled trial. JAMA, 304(5), 527-535.

Wells, S., Graham, K., Speechley, M., Koval, J.J. (2005). Drinking patterns, drinking contexts and alcohol-related aggression among late adolescent and young adult drinkers. Addiction 100(7): 933-944.

Wood, S. (2006). Generalized additive models: an introduction with R: CRC press.

Wood, S., \& Wood, M. (2016, March 3). Package MGCV. from https://cran.r-project.org/web/packages/mgcv/mgcv.pdf 2

Wood, S. N. (2012). On p-values for smooth components of an extended generalized additive model. Biometrika, 1-8.

Wright, E. M., Fagan, A. A., \& Pinchevsky, G. M. (2013). The effects of exposure to violence and victimization across life domains on adolescent substance use. Child Abuse Negl, 37(11), 899-909. doi: 10.1016/j.chiabu.2013.04.010

Zimmerman, M. A., Caldwell, C. H., \& Bernat, D. H. (2002). Discrepancy Between Self-Report and School-Record Grade Point Average: Correlates With Psychosocial Outcomes Among African American Adolescents1. Journal of Applied Social Psychology, 32(1), 86-109.

This article is protected by copyright. All rights reserved 